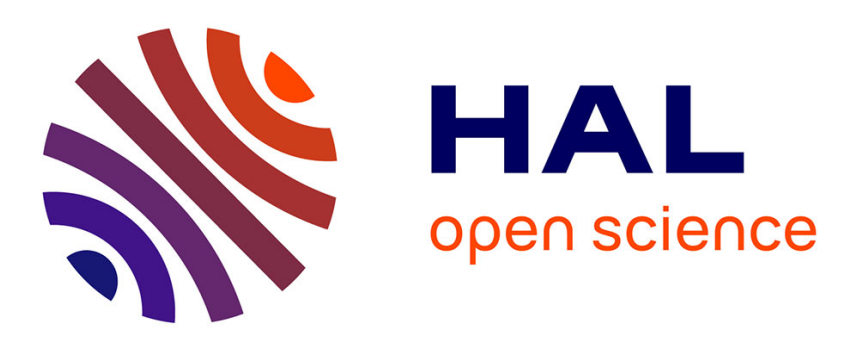

\title{
Sustainability of anaesthesia components of an enhanced recovery program (ERP) in colorectal and orthopaedics surgery
}

\author{
Hélène Beloeil, Karem Slim
}

\section{- To cite this version:}

Hélène Beloeil, Karem Slim. Sustainability of anaesthesia components of an enhanced recovery program (ERP) in colorectal and orthopaedics surgery. Anaesthesia Critical Care \& Pain Medicine, 2019, 38 (1), pp.25-28. 10.1016/j.accpm.2018.01.005 . hal-01811045

HAL Id: hal-01811045

https://hal-univ-rennes1.archives-ouvertes.fr/hal-01811045

Submitted on 5 Jul 2018

HAL is a multi-disciplinary open access archive for the deposit and dissemination of scientific research documents, whether they are published or not. The documents may come from teaching and research institutions in France or abroad, or from public or private research centers.
L'archive ouverte pluridisciplinaire HAL, est destinée au dépôt et à la diffusion de documents scientifiques de niveau recherche, publiés ou non, émanant des établissements d'enseignement et de recherche français ou étrangers, des laboratoires publics ou privés. 


\section{First page}

Sustainability of anaesthesia components of an enhanced recovery program (ERP) in colorectal and orthopaedics surgery

Helene Beloeil ${ }^{1}$, Karem Slim ${ }^{2}$, and the Francophone Group of enhanced recovery after surgery ${ }^{3}$

1 CHU Rennes, Pôle Anesthésie et Réanimation, Inserm, unité Numecan, CIC 1414 and Université de Rennes 1, F-35033 Rennes, France

2 CHU Clermont-Ferrand, Department of Digestive Surgery, Place Lucie Aubrac, 63000 ClermontFerrand, France

3 Grace, 9 allée du Riboulet, Beaumont, France

Address correspondence to Prof. Hélène Beloeil, Pôle d'Anesthésie Réanimation Chirurgicale, CHU

Rennes, 2 Rue Henri Le Guilloux, 35033 Rennes Cedex 9, France.

Phone: + 33299282422

Fax: + 33299282421

E-mail: helene.beloeil@chu-rennes.fr

\section{Conflicts of interest: none}

Funding: Funding was solely institutional.

Short title: Sustainability of ERP

Key words: ERP, sustainability, colorectal surgery, orthopaedics surgery

Sustainability of anaesthesia components of an enhanced recovery program (ERP) in colorectal and orthopaedics surgery

Helene Beloeil ${ }^{1}$, Karem Slim ${ }^{2}$, and the Francophone Group of enhanced recovery after surgery ${ }^{3}$

1 CHU Rennes, Department of anaesthesia and intensive care, Inserm, unité Numecan, CIC 1414 and Université de Rennes 1, F-35033 Rennes, France 
2 CHU Clermont-Ferrand, Department of Digestive Surgery, Place Lucie Aubrac, 63000 ClermontFerrand, France

3 Grace, 9 allée du Riboulet, Beaumont, France

Corresponding author: Prof. Hélène Beloeil, Department of Anaesthesia and surgical intensive care, CHU Rennes, 2 Rue Henri Le Guilloux, 35033 Rennes Cedex 9, France.

Phone: + 33299282422

Fax: + 33299282421

E-mail: helene.beloeil@chu-rennes.fr

\section{Conflicts of interest: none}

Funding: Funding was solely institutional.

Short title: Sustainability of ERP

Key words: ERP, sustainability, colorectal surgery; orthopaedics surgery

Sustainability of anaesthesia components of an enhanced recovery program (ERP) in colorectal and orthopaedics surgery 


\title{
Conflicts of interest: none
}

Funding: Funding was solely institutional.

Short title: Sustainability of ERP

Key words: ERP, sustainability, colorectal surgery, orthopaedics surgery

\begin{abstract}
Background: Sustainability of ERP is a challenge and data are scarce on the subject. The aim of this study was to assess if application of enhanced recovery elements through the Francophone Group of Enhanced Recovery after Surgery (Grace) in the anaesthesia management was sustainable 2 years after its implementation.
\end{abstract}

Materials and methods: We conducted a retrospective analysis of the prospective Grace database between October 2014 and October 2016. The evolution of each recommendation item over time was analysed using non-parametric Spearman correlation coefficient.

Results: A total of 67 and 43 centres corresponding to 2067 and 3022 patients participated to the Grace audit in colorectal and orthopaedics surgery, respectively. Colorectal surgery: Mean length of stay was $5( \pm 4)$ days and readmission rate was $6.6 \%$. Application of most items did not statistically 
change. It worsened over time for PONV prophylaxis $(\mathrm{p}=0.01)$ and prevention of intraoperative hypothermia $(\mathrm{p}=0.02)$; and improved for NSAID administration $(\mathrm{p}=0.01)$. Orthopaedics surgery: Mean length of stay was $3( \pm 2)$ days and readmission rate was $1.7 \%$. There was a trend towards improvement for most items. It reached statistical significance for PONV prophylaxis $(p=0.001)$, limited preoperative fasting $(p=0.01)$. While the use of a perineural catheter $(p=0.001)$ decreased over time, infiltration of the surgical site statistically increased $(\mathrm{p}=0.05)$.

Conclusion: This study shows on a large scale a trend towards less application of all ERP items over time. Continuous audits should be encouraged to expect further improvements.

\section{Introduction}

The benefits of enhanced recovery programs (ERP) have been widely demonstrated in the literature. ERP typically include items such as PONV prophylaxis, prevention of intraoperative hypothermia, limited preoperative fasting, multimodal analgesia etc... [1, 2]. Application of ERP after surgery leads to fewer morbidity and shorten hospital stay [3]. Methods to implement ERP in the daily practice have also been published by different groups, i.e. ERAS. The program of the Francophone group of enhanced recovery after surgery (Grace) program includes about 20 evidence-based care elements to reduce surgical stress (www.grace-asso.fr). Grace is a multidisciplinary group created in 2014 with the goal of enhancing the development of ERP [4]. Grace-certified centres voluntarily participate to on online prospective audit. Results published in 2017 , reported enhanced recovery and a decrease in hospital length of stay (LOS) to 6.5 days for colonic surgery and 3.4 days for orthopaedic surgery [4]. However, previous studies showing the benefit of ERP compared conventional with enhanced programs focused only on short-term results. After implementation of new guidelines has succeeded 
there is often a tendency for relapse into old routines after the initial implementation activities have ended [5]. When comparing with evidence-based guidelines which have always been poorly applied [6], one can hypothesise that application of ERP could not be sustained over time. Furthermore, data are scarce on the sustainability of the implementation of ERP over time [7, 8].

The aim of the present study was to assess if the application of enhanced recovery elements in the anaesthesia management was sustainable 2 years after its implementation in colorectal and orthopaedics surgery.

\section{Methods}

This is a retrospective analysis of a prospective database from the Francophone Group of Enhanced Recovery after Surgery (Groupe francophone de Réhabilitation Améliorée après Chirurgie - GRACE). The database named «Grace-Audit» is an online web-based one initiated in September 2014 after approval from the National Commission of Informatics and Liberties (CNIL) (no 1817711). For security reasons, the Grace-Audit records are hosted by the Sigma Datacentre (certified by the French Ministry for Health for health data storage) with a temporary password for each single access. The database is both a registry and an audit system. Data are collected by dedicated nurses or physicians in each "Grace" centre. Each patient's record contains approximately 100 variables regarding the hospital stay and discharge.

Orthopaedic surgery includes hip and knee surgeries. The following anaesthetic items are part of the ERP in colorectal and orthopaedic surgery: PONV prophylaxis, prevention of intraoperative hypothermia, preoperative fasting limited to 6 hours for solids and 2 hours for liquids, multimodal analgesia (more than two analgesia techniques), intraoperative goal-directed fluid therapy (GDFT), preoperative oral carbohydrates administration, NSAID administration for 48hours after surgery. In orthopaedic ERP, the following specific items are added: use of infiltration of the surgical site, use of perineural catheter. The aim of the study was to analyse the sustainability of the application by anaesthetists of these ERP items between October 2014 and October 2016. Sustainability was defined as a non-statistically significant decrease of the use of the selected items. 


\section{Statistics}

Statistical analysis was performed using Stata 13 software (StataCorp LP, College Station, TX, US). Quantitative data were presented as mean ( \pm standard-deviation) or median [interquartile range], according to statistical distribution (assumption of normality assessed using the Shapiro-Wilk test) and as frequencies and associated percentages for categorical parameters. The study of the evolution of each recommendation item over time was analysed using non-parametric Spearman correlation coefficient. The tests were two-sided, with a type I error set at $\alpha=0.05$.

\section{Results}

Between October 2014 and October 2016, a total of 67 and 43 centres corresponding to 2067 and 3022 patients participated to the Grace-audit in colorectal surgery and in orthopaedics, respectively. The centres involved were private hospitals $(n=30 / 43$ for orthopaedics and $n=26 / 67$ for colorectal surgery), general hospitals ( $n=9 / 43$ for orthopaedics and $n=25 / 67$ for colorectal surgery) and teaching hospitals ( $n=4 / 43$ for orthopaedics and $n=16 / 67$ for colorectal surgery).

\section{Colorectal surgery}

During the study period, mean length of stay was $5( \pm 4)$ days and readmission rate was $6.6 \%$ after colorectal surgery. Application of most enhanced recovery items did not statistically change and remained above $80 \%$ for limited preoperative fasting $(\mathrm{p}=0.78)$ and multimodal analgesia $(\mathrm{p}=0.49)$. Although not statistically significant, it decreased from $100 \%$ to $43 \%$ for GDFT $(\mathrm{p}=0.07$ ) (figure 1). Application significantly worsened over time for 2 items: PONV prophylaxis $(\mathrm{p}=0.01)$ and prevention of intraoperative hypothermia $(\mathrm{p}=0.02)$. The only item for which application improved over time was NSAID administration $(\mathrm{p}=0.01)$.

\section{Orthopaedic surgery}

During the study period, mean length of stay was $3( \pm 2)$ days and readmission rate was $1.7 \%$ after orthopaedic surgery. There was a trend towards improvement for most enhanced recovery items. It did not reached statistical significance for intraoperative hypothermia prevention $(p=0.14)$, GDFT $(p=$ 0.23 ) and oral carbohydrates administration ( $\mathrm{p}=0.53$ ), (Figure 2). It significantly improved for PONV 
prophylaxis $(p=0.001)$ and limited preoperative fasting $(p=0.01)$. Specific items on perioperative analgesia in orthopaedic are presented figure 3 . While the use of a perineural catheter $(\mathrm{p}=0.001)$ decreased over time, infiltration of the surgical site statistically increased $(p=0.05)$. Administration of multimodal analgesia $(\mathrm{p}=0.18)$ was stable while NSAID administration $(\mathrm{p}=0.02)$ decreased over time.

\section{Discussion}

The results of this study, which included more than 2000 patients in colorectal surgery and more than 3000 in orthopaedics surgery, show a clear trend towards less application of all ERP items over time (implementation effect) though given the sample size this may not be statistically significant

\section{Colorectal surgery}

Our results are somewhat in accordance with those of two smaller studies from the Netherlands [7] and Switzerland [8]. The Dutch study showed a slight decrease in protocol adherence but the main result was the variation between the hospitals in term of sustainability [7]. The Swiss study included relatively few patients $(n=482)$ and showed the same decrease in overall adherence with the program from $77 \%$ in 2013 to $73 \%$ in 2014 with no significant impact on the length of stay [8]. Regarding the present study, at the time of the implementation in 2014, all centres followed the ERP for most items. There was a clear trend towards less application of all ERP items with time. In particular, it significantly deteriorated for PONV prophylaxis, hypothermia prevention and GDFT (although not statistically significant for the latest item). There is probably "an implementation effect" like the Hawthorn effect in clinical research. Indeed, at the time of the implementation of a new paradigm, medical and paramedical teams are focused on the new program and all centres were applying most items in our study. Similarly to other studies application rates dropped after. Teams tend to return to the previous routine $[7,9]$. It remains unclear why PONV prophylaxis, hypothermia prevention and GDFT were the least sustainable items. These items are highly dependent on the physician's prescription. Despite all the literature and the proofs of the deleterious effect of hypothermia, its prevention is still not part of the standard of care in most centres [10]. Routine practice is to apply the warming device after the induction of anaesthesia. Sun et al. [11] demonstrated that it is already too 
late and this practice leads to hypothermia in the first hour. Active warming devices should be applied preoperatively. Similarly, despite a widely demonstrated evidence-based benefit GDFT is not reinforced by physicians [12]. It necessitates an active participation of the anaesthesiologist in charge and is often accused of being time-consuming.

The only 2 items for which we observed a significant improvement in the implementation over time were 48 hours NSAID prescription and preoperative oral carbohydrates administration. However, it was not difficult to improve, as the level of adherence to these 2 items was very low at the time of implementation. Moreover, the publication of recommendations on NSAID administration in colorectal surgery has probably helped improving the prescription [1]. Indeed, the controversy on the potential harm associated with NSAID in colorectal surgery was at its peak in 2014. Articles published at the time suggested that NSAID could increase anastomosis leakage $[13,14]$. Since then, the analysis of the literature led to recommendations stating that NSAID are probably recommended after colorectal surgery as a component of multimodal analgesia in patients without any risk factors of anastomotic leak [15].

\section{Orthopaedic surgery}

At the time of the implementation in 2014, the ERP was applied by less than $50 \%$ of the centres. Then, adherence to all items significantly increased over the next two years. The rise of the infiltration of the surgical site was associated with the fall of the use of perineural catheter and NSAID. This evolution corresponds to the general evolution of both practice and literature [16, 17]. More and more centres have switched from peripheral nerve block and/or catheter to an infiltration of the site with a mixture containing local anaesthetics and NSAID $[18,19]$.

Our results are in accordance with the literature. ERP adherence usually drops after the implementation phase [7]. A parallel can be drawn with the low adherence to guidelines published in the literature [20, 21]. A traditional inconsistency between guidelines and practice [6] as well as an inefficacy of the implementation strategy developed following publication of guidelines has been described. Despite quality processes involving continuing medical evaluation, the adherence rate stays low [22]. Sustaining ERP is indeed probably the most challenging part of ERP! Improvement can only 
be expected by implementing multiples strategies focusing on organization, patients and professionals' active participation. Possible organizational modifications to improve protocol adherence include a profound reorganization of the ward. Care elements (mobilisation of patients, meals in a common room not in the patient's room...etc.) should be part of the structure therefore return to old routine is not possible [23]. Such rehabilitation wards have to be specially designed. Patients also need to be involved. To ensure success, some authors proposed self-completed recovery diaries as a method to encourage patients to keep track of their own recovery process and to meet protocol targets [24]. However, as the present study shows the barriers to actually implement ERPs are not mainly related to the patients' perceptions [25]. With regard to professionals, regular information about performance is a keystone to sustainability [26]. English experts even recommended to create a national network to spread the best practices [27]. In our opinion, beyond any evidence-based recommendations, continuous audit systems can also help the professionals to self-audit their practice. With the data in hands the leaders or the dedicated professionals should organize regular meetings with the whole team to improve the practices and therefore to overcome the barriers of sustainability of ERPs. For this purpose, and like others, our group has developed such an audit-system and is providing it freely for our francophone colleagues [28].

\section{Conclusion}

This study shows on a large scale (over 5000 patients) a trend towards less application of all ERP items over time. Results varied amongst items. Future efforts should focus and PONV prophylaxis, hypothermia prevention and GDFT during colorectal surgery. Moreover, continuous audit systems should be encouraged to expect further improvements.

Acknowledgements: We thank Mr Bruno Pereira for his assistance in the statistical analyses and all the participants to the database Grace-Audit for their valuable work 


\section{Legend of the figures:}

Figure 1: Evolution of anaesthesia elements in colorectal surgery. Data are expressed in \%. Oral CH: preoperative oral carbohydrates; Limited fasting: preoperative fasting limited to 6 hours; GDFT: intraoperative goal-directed fluid therapy; PONV: postoperative nausea and vomiting; NSAID: nonsteroidal anti-inflammatory drugs.

Figure 2: Evolution of anaesthesia elements in orthopaedics. Data are expressed in \%. Tobacco and alcohol: withdrawal; Oral CH: preoperative oral carbohydrates; Limited fasting: preoperative fasting limited to 6 hours; GDFT: intraoperative goal-directed fluid therapy; PONV: postoperative nausea and vomiting.

Figure 3: Evolution of analgesia elements in orthopaedics. Data are expressed in \%. Infiltration: periarticular infiltration of the surgical site; Analgesia catheter: perineural catheter for postoperative analgesia; NSAID: non-steroidal anti-inflammatory drugs.

\section{References}

Alfonsi P, Slim K, Chauvin $\mathrm{M}$ et al. French guidelines for enhanced recovery after elective colorectal surgery. Journal of visceral surgery 2014; 151: 65-79. 
Kehlet $\mathrm{H}$ and Thienpont E. Fast-track knee arthroplasty -- status and future challenges. The Knee 2013; 20 Suppl 1: S29-33.

Zhao JH, Sun JX, Gao P et al. Fast-track surgery versus traditional perioperative care in laparoscopic colorectal cancer surgery: a meta-analysis. BMC cancer 2014; 14: 607.

Veziant J, Raspado O, Entremont A et al. Large-scale implementation of enhanced recovery programs after surgery. A francophone experience. Journal of visceral surgery 2017; 154: 159-166.

Schell SF, Luke DA, Schooley MW et al. Public health program capacity for sustainability: a new framework. Implementation science : IS 2013; 8: 15.

Bryson GL, Wyand A and Bragg PR. Preoperative testing is inconsistent with published guidelines and rarely changes management. Canadian journal of anaesthesia = Journal canadien d'anesthesie 2006; 53: 236-241.

Gillissen F, Ament SM, Maessen JM et al. Sustainability of an enhanced recovery after surgery program (ERAS) in colonic surgery. World journal of surgery 2015; 39: 526-533.

Martin D, Roulin D, Addor V, Blanc C, Demartines N and Hubner M. Enhanced recovery implementation in colorectal surgery-temporary or persistent improvement? Langenbeck's archives of surgery 2016; 401: 11631169 .

Hendry PO, Hausel J, Nygren J et al. Determinants of outcome after colorectal resection within an enhanced recovery programme. Br J Surg 2009; 96: 197-205.

Torossian A and Group TS. Survey on intraoperative temperature management in Europe. Eur J Anaesthesiol 2007; 24: 668-675.

Sun Z, Honar H, Sessler DI et al. Intraoperative core temperature patterns, transfusion requirement, and hospital duration in patients warmed with forced air. Anesthesiology 2015; 122: 276-285.

Thiele RH, Raghunathan K, Brudney CS et al. American Society for Enhanced Recovery (ASER) and Perioperative Quality Initiative (POQI) joint consensus statement on perioperative fluid management within an enhanced recovery pathway for colorectal surgery. Perioperative medicine 2016; 5: 24.

Klein M, Gogenur I and Rosenberg J. Postoperative use of non-steroidal anti-inflammatory drugs in patients with anastomotic leakage requiring reoperation after colorectal resection: cohort study based on prospective data. BMJ 2012; 345: e6166.

Burton TP, Mittal A and Soop M. Nonsteroidal anti-inflammatory drugs and anastomotic dehiscence in bowel surgery: systematic review and meta-analysis of randomized, controlled trials. Diseases of the colon and rectum 2013; 56: 126-134.

Slim K, Joris J, Beloeil H and Groupe Francophone de Rehabilitation Amelioree apres C. Colonic anastomoses and non-steroidal anti-inflammatory drugs. Journal of visceral surgery 2016; 153: 269-275.

Husted H. Fast-track hip and knee arthroplasty: clinical and organizational aspects. Acta orthopaedica Supplementum 2012; 83: 1-39.

Khan SK, Malviya A, Muller SD et al. Reduced short-term complications and mortality following Enhanced Recovery primary hip and knee arthroplasty: results from 6,000 consecutive procedures. Acta orthopaedica 2014; 85: $26-31$.

Specht K, Leonhardt JS, Revald P et al. No evidence of a clinically important effect of adding local infusion analgesia administrated through a catheter in pain treatment after total hip arthroplasty. Acta orthopaedica 2011; 82: $315-320$.

Andersen LO and Kehlet H. Analgesic efficacy of local infiltration analgesia in hip and knee arthroplasty: a systematic review. Br J Anaesth 2014; 113: 360-374.

Chapman SA, St Hill CA, Little MM et al. Adherence to treatment guidelines: the association between stroke risk stratified comparing CHADS2 and CHA2DS2-VASc score levels and warfarin prescription for adult patients with atrial fibrillation. BMC health services research 2017; 17: 127.

Cabana MD, Rand CS, Powe NR et al. Why don't physicians follow clinical practice guidelines? A framework for improvement. Jama 1999; 282: 1458-1465.

Sigmund AE, Stevens ER, Blitz JD and Ladapo JA. Use of Preoperative Testing and Physicians' Response to Professional Society Guidance. JAMA internal medicine 2015; 175: 1352-1359.

Maessen J, Dejong CH, Hausel J et al. A protocol is not enough to implement an enhanced recovery programme for colorectal resection. Br J Surg 2007; 94: 224-231.

Hulscher ME, Schouten LM, Grol RP and Buchan H. Determinants of success of quality improvement collaboratives: what does the literature show? BMJ quality \& safety 2013; 22: 19-31.

Alawadi ZM, Leal I, Phatak UR et al. Facilitators and barriers of implementing enhanced recovery in colorectal surgery at a safety net hospital: A provider and patient perspective. Surgery 2016; 159: 700-712.

Ament SM, Gillissen F, Moser A et al. Identification of promising strategies to sustain improvements in hospital practice: a qualitative case study. BMC health services research 2014; 14: 641.

Knott P, Lonner B, Smith M, Frommeyer E and Ren Y. Measuring anterior trunk deformity in scoliosis: development of asymmetry parameters using surface topography (a pilot study). Scoliosis and spinal disorders 2016; 11: 32 . 
Slim K, Delaunay L, Joris J et al. How to implement an enhanced recovery program? Proposals from the Francophone Group for enhanced recovery after surgery (GRACE). Journal of visceral surgery 2016; 153: S45S49.

We wish to confirm that there are no known conflicts of interest associated with this publication and there has been no significant financial support for this work that could have influenced its outcome.

We confirm that the manuscript has been read and approved by all named authors and that there are no other persons who satisfied the criteria for authorship but are not listed. We further confirm that the order of authors listed in the manuscript has been approved by all of us.

We confirm that we have given due consideration to the protection of intellectual property associated with this work and that there are no impediments to publication, including the timing of publication, with respect to intellectual property. In so doing we confirm that we have followed the regulations of our institutions concerning intellectual property.

We further confirm that any aspect of the work covered in this manuscript that has involved either experimental animals or human patients has been conducted with the ethical approval of all relevant bodies and that such approvals are acknowledged within the manuscript.

We understand that the Corresponding Author is the sole contact for the Editorial process (including Editorial Manager and direct communications with the office). He/she is responsible for communicating with the other authors about progress, submissions of revisions and final approval of proofs. We confirm that we have provided a current, correct email address which is accessible by the Corresponding Author and which has been configured to accept email from (helene.beloeil@churennes.fr)

Signed by all authors as follows:

Helene Beloeil

Karem Slim 


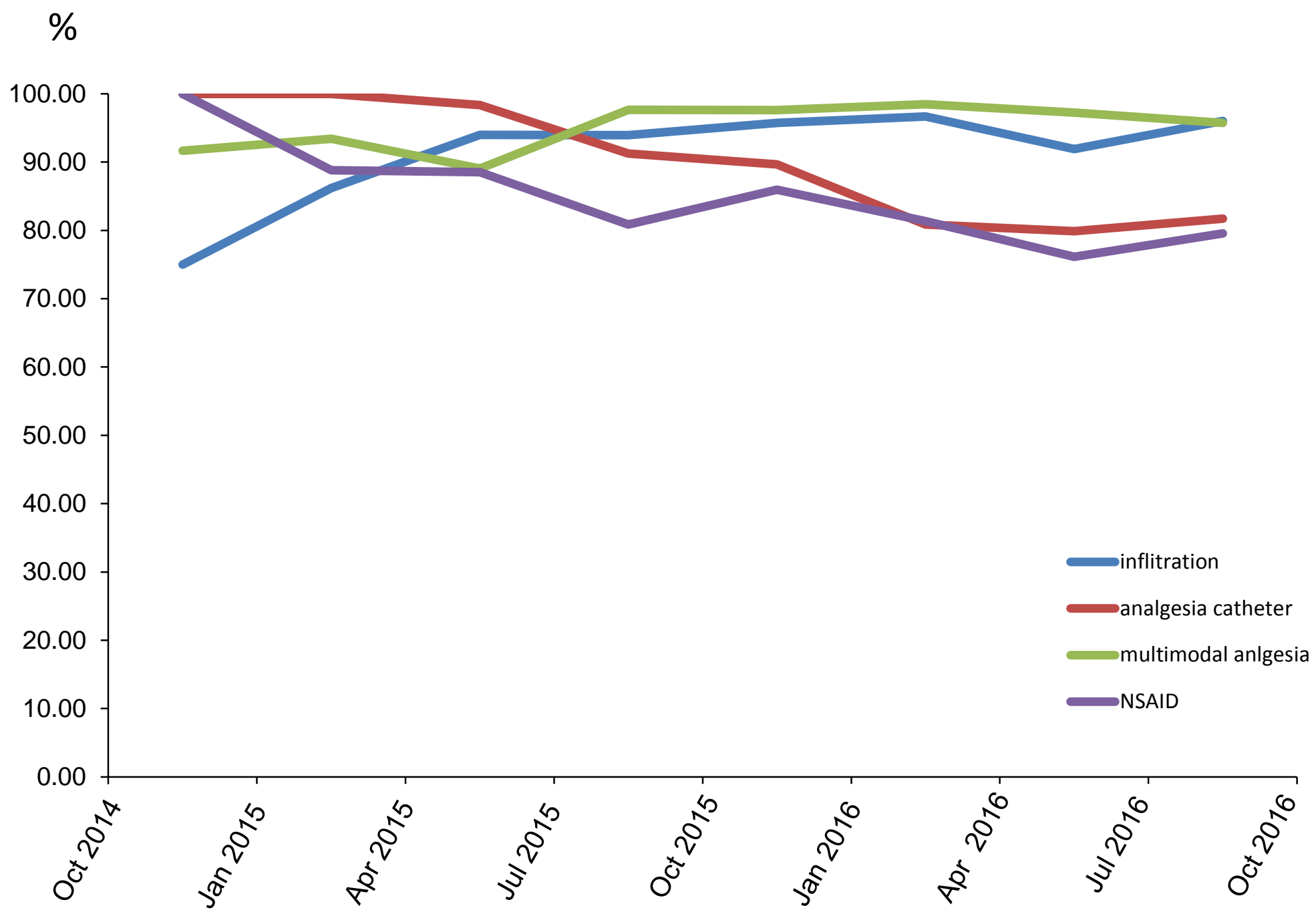




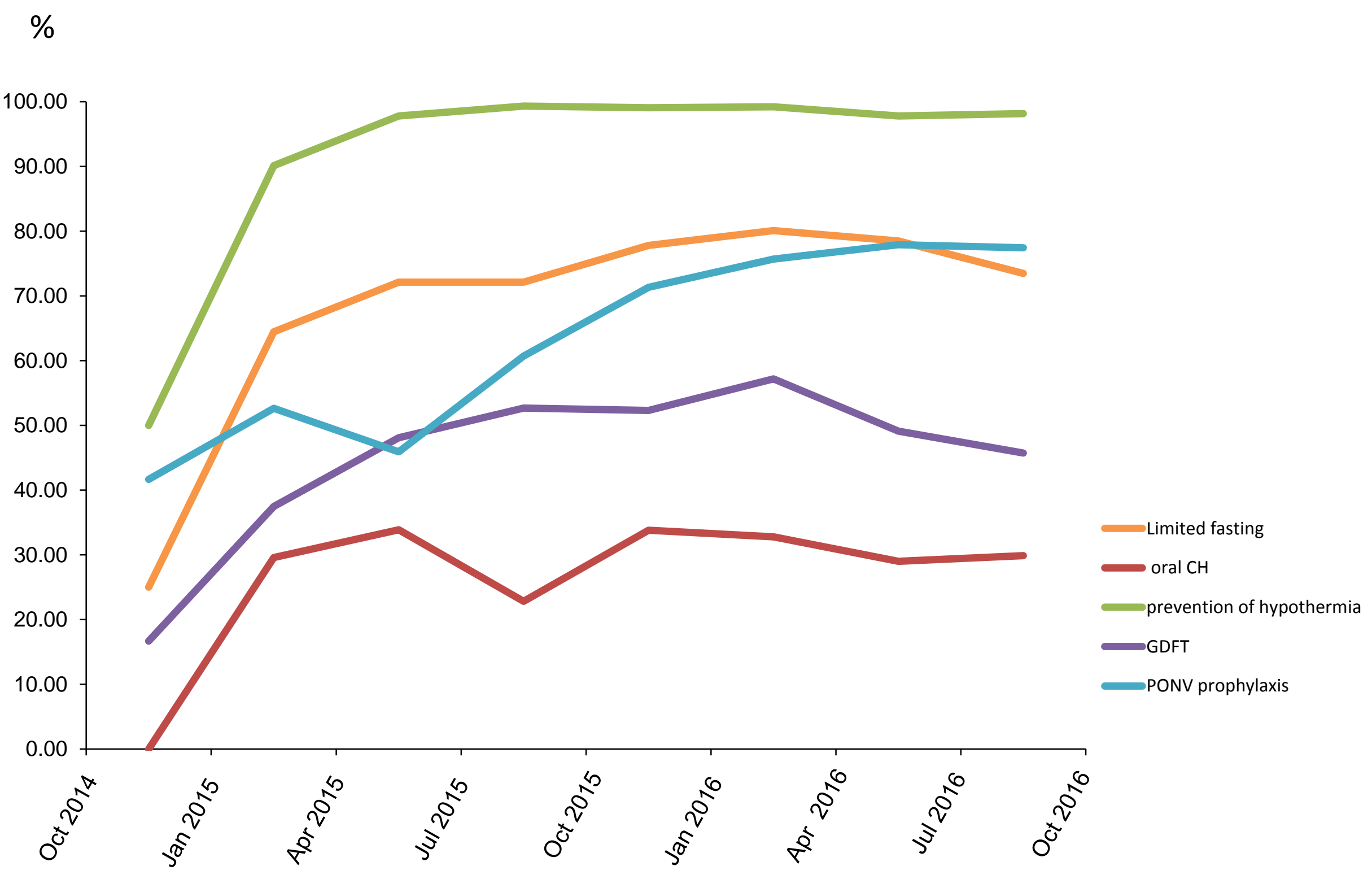

Fig 2 
